\title{
$7 \mathrm{keV}$ sterile neutrino dark matter in $\mathrm{U}(1)_{R^{-l e p t o n}}$ number model
}

\section{Sabyasachi Chakraborty, Dilip Kumar Ghosh and Sourov Roy}

Department of Theoretical Physics, Indian Association for the Cultivation of Science, 2A \& 2B Raja S.C.Mullick Road, Jadavpur, Kolkata 700 032, India

E-mail: tpsc3@iacs.res.in, tpdkg@iacs.res.in, tpsr@iacs.res.in

ABSTRACT: We study the phenomenology of a $\mathrm{keV}$ sterile neutrino in a supersymmetric model with $\mathrm{U}(1)_{R}$-lepton number in the light of a very recent observation of an X-ray line signal at around $3.5 \mathrm{keV}$, detected in the X-ray spectra of Andromeda galaxy and various galaxy clusters including the Perseus galaxy cluster. This model not only provides a small tree level mass to one of the active neutrinos but also renders a suitable warm dark matter candidate in the form of a sterile neutrino with negligible active-sterile mixing. Light neutrino masses and mixing can be explained once one-loop radiative corrections are taken into account. The scalar sector of this model can accommodate a Higgs boson with a mass of $\sim 125 \mathrm{GeV}$. In this model gravitino is the lightest supersymmetric particle (LSP) and we also study the cosmological implications of this light gravitino with mass $\sim \mathcal{O}(\mathrm{GeV})$.

KeYwords: Supersymmetry Phenomenology

ARXIV EPRINT: 1405.6967 


\section{Contents}

1 Introduction 1

$2 \mathrm{U}(1)_{R^{-}}$-lepton number model with a right handed neutrino superfield $\quad 3$

3 Scalar sector $\quad 5$

$4 \quad$ R-symmetry breaking $\quad 6$

5 Right handed neutrino as a keV warm dark matter 9

$\begin{array}{lll}5.1 & \text { Sterile neutrino decay } & 12\end{array}$

6 Gravitino cosmology 13

$\begin{array}{lll}7 & \text { Conclusion } & 14\end{array}$

\section{Introduction}

We are living in an era enriched with many experimental breakthroughs and results especially in the area of astro-particle physics and cosmology. The most recent one is the identification of a weak line at $E \sim 3.5 \mathrm{keV}$ in the X-ray spectra of the Andromeda galaxy and many other galaxy clusters including the Perseus galaxy cluster, observed by XMMNewton X-ray Space observatory [1,2]. The observed flux and the best fit energy peak are at

$$
\begin{aligned}
& \Phi_{\gamma}=4 \pm 0.8 \times 10^{-6} \text { photons } \mathrm{cm}^{-2} \mathrm{sec}^{-1} \\
& E_{\gamma}=3.57 \pm 0.02 \mathrm{keV} .
\end{aligned}
$$

Since atomic transitions in thermal plasma cannot account for this energy, therefore the concept of a dark matter, providing the possible explanation regarding the appearance of this photon line becomes extremely important. This result can be explained by a sterile neutrino [3-8], axion or axion like warm dark matter [9-12], axino [13-15], excited dark matter [16, 17], gravitino [18, 19] and keV scale LSP [20] as decaying dark matter. Other interesting scenarios with an annihilating scalar dark matter [21], decaying Majoron [22] and a keV scale dark gaugino [23] have also been considered in this context. In this work we consider sterile neutrino in a $\mathrm{U}(1)_{R}$-lepton number model, which could provide a possible explanation for the emergence of the photon line. The observed flux and the peak of the energy readily translates to an active-sterile mixing in the range $2.2 \times 10^{-11}<\sin ^{2} 2 \theta_{14}<$ $2 \times 10^{-10}$ and the mass of the sterile neutrino dark matter $M_{N}^{R}=7.06 \pm 0.05 \mathrm{keV}[2]$.

On the other hand, in high energy collider frontier two CERN based experiments ATLAS and CMS have confirmed the existence of a neutral elementary scalar boson of 
nature, with mass around $125 \mathrm{GeV}[24,25]$. Nevertheless, more analysis is required to confirm it as the Standard Model (SM) Higgs boson. In order to explain the mass of this scalar boson in a natural way, to address the question of nonzero neutrino mass and mixing and to provide a candidate for dark matter, many beyond standard model (BSM) theories have been pursued for quite some time and supersymmetry remains one of the most celebrated ones as of now. However, supersymmetric particle searches by ATLAS and CMS experiments for pp collision at center of mass energy 7 and $8 \mathrm{TeV}$, have observed no significant excess $[26,27]$ over the standard model background. This has put very stringent lower limits on the superpartner masses.

In the light of this present situation, $\mathrm{U}(1)_{R}$-symmetric models with Dirac gauginos are well motivated because they can relax the strong bounds on the superpartner masses, explain the $125 \mathrm{GeV}$ Higgs boson mass, provide non-zero neutrino mass at the tree as well as at the one loop level and can also accommodate a suitable dark matter candidate. Various aspects of different R-symmetric models have been studied and can be found in the literature [28-53]. In this work, we study a particular $\mathrm{U}(1)_{R^{-}}$-symmetric model where we have identified the R-charges with lepton numbers in such a way that the lepton numbers of the standard model fermions correspond to the negative of their R-charges [48, 49]. The role of the down-type Higgs is played by the sneutrino since its vacuum expectation value (vev) is not constrained by the Majorana mass of the neutrino. The minimal extension of this model by adding a single right handed neutrino superfield also gives rise to very interesting phenomenological consequences [50]. It generates a tree level Dirac mass for one of the neutrinos in the R-symmetry preserving scenario. If R-symmetry is broken because of the presence of a non zero gravitino mass, then for small neutrino Yukawa coupling, $f \sim \mathcal{O}\left(10^{-4}\right)$, the extended neutralino-neutrino mass matrix provides a sterile neutrino state accompanied by an active neutrino state. Here we identify the sterile neutrino as the warm dark matter in our model.

The presence of R-symmetry inhibits gauginos to acquire a Majorana mass. However, gauginos can have Dirac masses and to introduce the Dirac gaugino mass, one must consider a singlet chiral superfield $\hat{S}$, a triplet $\hat{T}$ and an octet $\hat{O}$ living in the adjoint representation of $\mathrm{U}(1)_{Y}, \mathrm{SU}(2)_{L}$ and $\mathrm{SU}(3)_{C}$ respectively. The Dirac gaugino masses are also coined as 'supersoft' mass terms since they do not contribute to any logarithmic corrections to the scalar masses. The presence of Dirac gluino also helps to relax the bound on squark masses compared to MSSM and in addition flavor and CP violating constraints are suppressed in this class of models [35].

The plan of the paper is as follows. At first we describe the model in section 2, with appropriate R-charge assignments. In section 3 we discuss very briefly, the scalar sector of the model and point out the extra contributions to the Higgs boson mass, which can arise both at the tree level as well as at the one loop level. Section 4 addresses the issue of R-symmetry breaking and tree level Majorana masses of the sterile and one of the active neutrinos. Next in section 5 the essential features of the sterile neutrino as a $\mathrm{keV}$ warm dark matter candidate are discussed and its production mechanism and the dominant decay modes relevant to our model are highlighted. In section 6 we briefly present a discussion related to the cosmology of the gravitino in this model with a few $\mathrm{GeV}$ mass and finally, in section 7 , we summarise our results. 


\begin{tabular}{|c|ccccccccccccc|}
\hline & $\hat{Q}_{i}$ & $\hat{U}_{i}^{c}$ & $\hat{D}_{i}^{c}$ & $\hat{L}_{i}$ & $\hat{E}_{i}^{c}$ & $\hat{H}_{u}$ & $\hat{H}_{d}$ & $\hat{R}_{u}$ & $\hat{R}_{d}$ & $\hat{S}$ & $\hat{T}$ & $\hat{O}$ & $\hat{N}^{c}$ \\
\hline $\mathrm{U}(1)_{R}$ & 1 & 1 & 1 & 0 & 2 & 0 & 0 & 2 & 2 & 0 & 0 & 0 & 2 \\
\hline
\end{tabular}

Table 1. $\mathrm{U}(1)_{R}$ charge assignments of the chiral superfields.

\section{$2 \mathrm{U}(1)_{R^{-}}$lepton number model with a right handed neutrino superfield}

We study a $\mathrm{U}(1)_{R}$-lepton number model, where in addition to the standard superfields of the MSSM - $\hat{H}_{u}, \hat{H}_{d}, \hat{Q}_{i}, \hat{U}_{i}{ }^{c}, \hat{D}_{i}{ }^{c}, \hat{L}_{i}, \hat{E}_{i}{ }^{c}$, this model includes a right handed neutrino superfield and a pair of vector-like $\mathrm{SU}(2)_{L}$ doublet superfields $\hat{R}_{u}$ and $\hat{R}_{d}$, with opposite hypercharge [50]. These two doublets carry non zero R-charges (The R-charge assignments are given in table 1) and therefore, to avoid spontaneous R-breaking and the emergence of R-axions, they do not acquire any non-zero vev and would remain inert. $\mathrm{R}$ symmetry prohibits soft supersymmetry breaking terms like Majorana gaugino masses and trilinear scalar couplings. However, gauginos can acquire Dirac masses as mentioned in the introduction. The implications of adding a right-handed neutrino superfield $\hat{N}^{c}$ is discussed later in detail. We would like to reiterate that the R-charge assignments are such that the lepton number of the SM fermions are negative of their corresponding R-charges.

The generic superpotential, carrying R-charge of 2 units is

$$
\begin{aligned}
W= & y_{i j}^{u} \hat{H}_{u} \hat{Q}_{i} \hat{U}_{j}^{c}+\mu_{u} \hat{H}_{u} \hat{R}_{d}+f_{i} \hat{L}_{i} \hat{H}_{u} \hat{N}^{c}+\lambda_{S} \hat{S} \hat{H}_{u} \hat{R}_{d}+2 \lambda_{T} \hat{H}_{u} \hat{T} \hat{R}_{d}-M_{R} \hat{N}^{c} \hat{S} \\
& +\mu_{d} \hat{R}_{u} \hat{H}_{d}+\lambda_{S}^{\prime} \hat{S} \hat{R}_{u} \hat{H}_{d}+\frac{1}{2} \lambda_{i j k} \hat{L}_{i} \hat{L}_{j} \hat{E}_{k}^{c}+\lambda_{i j k}^{\prime} \hat{L}_{i} \hat{Q}_{j} \hat{D}_{k}^{c}+2 \lambda_{T}^{\prime} \hat{R}_{u} \hat{T} \hat{H}_{d} \\
& +y_{i j}^{d} \hat{H}_{d} \hat{Q}_{i} \hat{D}_{j}^{c}+y_{i j}^{l} \hat{H}_{d} \hat{L}_{i} \hat{E}_{j}^{c}+\lambda_{N} \hat{N}^{c} \hat{H}_{u} \hat{H}_{d} .
\end{aligned}
$$

Note that a subset $\left(\lambda, \lambda^{\prime}\right)$ of standard R-parity violating operators are present in the superpotential although the model is $\mathrm{U}(1)_{R}$ conserving (i.e. lepton number conserving). In a somewhat simplistic approach we have omitted the terms $\hat{N}^{c} \hat{S} \hat{S}$ and $\hat{N}^{c}$ from the superpotential.

In a realistic model one should also include supersymmetry breaking terms, such as the gaugino and scalar mass terms. The Dirac gaugino 'supersoft' mass terms are constructed from a spurion superfield $W_{\alpha}^{\prime}=\lambda_{\alpha}+\theta_{\alpha} D^{\prime}$, if supersymmetry breaking is of the D-type. The Lagrangian containing the Dirac gaugino masses are $[39,40]$

$$
\mathcal{L}_{\text {gaugino }}^{\text {Dirac }}=\int d^{2} \theta \frac{W_{\alpha}^{\prime}}{\Lambda}\left[\sqrt{2} \kappa_{1} W_{1 \alpha} \hat{S}+2 \sqrt{2} \kappa_{2} \operatorname{tr}\left(W_{2 \alpha} \hat{T}\right)+2 \sqrt{2} \kappa_{3} \operatorname{tr}\left(W_{3 \alpha} \hat{O}\right)\right]+\text { h.c. }
$$

This D-term breaking generates Dirac mass for the gauginos, proportional to $k_{i} \frac{<D^{\prime}>}{\Lambda}$, where $\Lambda$ denotes the scale of SUSY breaking mediation. In a similar manner the $\mathrm{U}(1)_{R}$ conserving soft supersymmetry breaking terms in the scalar sector are generated by the spurion superfield $\hat{X}$, defined as $\hat{X}=x+\theta^{2} F_{X}$. The non-zero vev of the F-term generates 
the scalar soft terms as

$$
\begin{aligned}
V_{\text {soft }}= & m_{H_{u}}^{2} H_{u}^{\dagger} H_{u}+m_{R_{u}}^{2} R_{u}^{\dagger} R_{u}+m_{H_{d}}^{2} H_{d}^{\dagger} H_{d}+m_{R_{d}}^{2} R_{d}^{\dagger} R_{d}+m_{\tilde{L}_{i}}^{2} \tilde{L}_{i}^{\dagger} \tilde{L}_{i} \\
& +m_{\tilde{R}_{i}}^{2} \tilde{l}_{R i}^{\dagger} \tilde{l}_{R i}+M_{N}^{2} \tilde{N}^{c \dagger} \tilde{N}^{c}+m_{S}^{2} S^{\dagger} S+2 m_{T}^{2} \operatorname{tr}\left(T^{\dagger} T\right)+2 m_{O}^{2} \operatorname{tr}\left(O^{\dagger} O\right) \\
& +\left(B \mu H_{u} H_{d}+\text { h.c. }\right)-\left(b \mu_{L}^{i} H_{u} \tilde{L}_{i}+\text { h.c. }\right)+\left(t_{S} S+\text { h.c. }\right) \\
& +\frac{1}{2} b_{S}\left(S^{2}+\text { h.c. }\right)+b_{T}(\operatorname{tr}(T T)+\text { h.c. })+B_{O}(\operatorname{tr}(O O)+\text { h.c. }) .
\end{aligned}
$$

The presence of the bilinear term $b \mu_{L}^{i} H_{u} \tilde{L}_{i}$ in the soft supersymmetry breaking potential implies all the three left handed sneutrinos can acquire non zero vevs $\left(v_{i}\right)$. To simplify, we perform a basis rotation as $\hat{L}_{i}=\frac{v_{i}}{v_{a}} \hat{L}_{a}+e_{i b} \hat{L}_{b}$ by which only one of the sneutrinos acquire a non zero vev $\left(v_{a}\right)$ and we choose it to be the electron sneutrino $(a=1(e))$. We also choose the neutrino Yukawa coupling $(f)$ in such a manner that only $\hat{L}_{a}$ couples with $\hat{N}^{c}$, the right-handed neutrino superfield [50]. Finally, we choose a very large $\mu_{d}$ such that the superfields $\hat{H}_{d}$ and $\hat{R}_{u}$ gets decoupled, which also implies that the left handed electron type sneutrino now plays the role of a down type Higgs field. We would like to emphasise that the model is lepton number conserving and therefore, the sneutrino vev is not constrained from the Majorana mass of the neutrinos. This is clearly different from the standard R-parity violating scenario.

In the mass eigenstate basis (primed superfields) of the down-type quarks and the charged leptons ${ }^{1}$ the superpotential takes the following form [50]

$$
W=y_{i j}^{u} \hat{H}_{u} \hat{Q}_{i} \hat{U}_{j}^{c}+\mu_{u} \hat{H}_{u} \hat{R}_{d}+f \hat{L}_{a} \hat{H}_{u} \hat{N}^{c}+\lambda_{S} \hat{S} \hat{H}_{u} \hat{R}_{d}+2 \lambda_{T} \hat{H}_{u} \hat{T} \hat{R}_{d}-M_{R} \hat{N}^{c} \hat{S}+W^{\prime}
$$

and

$$
\begin{aligned}
W^{\prime}= & \sum_{b=2,3} f_{b}^{l} \hat{L}_{a}^{\prime} \hat{L}_{b}^{\prime} \hat{E}_{b}^{\prime c}+\sum_{k=1,2,3} f_{k}^{d} \hat{L}_{a}^{\prime} \hat{Q}_{k}^{\prime} \hat{D}_{k}^{\prime c}+\sum_{k=1,2,3} \frac{1}{2} \tilde{\lambda}_{23 k} \hat{L}_{2}^{\prime} \hat{L}_{3}^{\prime} \hat{E}_{k}^{\prime c} \\
& +\sum_{j, k=1,2,3 ; b=2,3} \tilde{\lambda}_{b j k}^{\prime} \hat{L}_{b}^{\prime} \hat{Q}_{j}^{\prime} \hat{D}_{k}^{\prime c} .
\end{aligned}
$$

In our subsequent analysis we stay in this mass basis but remove the prime from the fields and make the replacement $\tilde{\lambda}, \tilde{\lambda}^{\prime} \rightarrow \lambda, \lambda^{\prime}$. The soft supersymmetry breaking but $\mathrm{U}(1)_{R}$ preserving terms in the rotated basis are

$$
\begin{aligned}
V_{\text {soft }}= & m_{H_{u}}^{2} H_{u}^{\dagger} H_{u}+m_{R_{d}}^{2} R_{d}^{\dagger} R_{d}+m_{\tilde{L}_{a}}^{2} \tilde{L}_{a}^{\dagger} \tilde{L}_{a}+\sum_{b=2,3} m_{\tilde{L}_{b}}^{2} \tilde{L}_{b}^{\dagger} \tilde{L}_{b}+M_{N}^{2} \tilde{N}^{c \dagger} \tilde{N}^{c}+m_{\tilde{R}_{i}}^{2} \tilde{l}_{R i}^{\dagger} \tilde{l}_{R i} \\
& +m_{S}^{2} S^{\dagger} S+2 m_{T}^{2} \operatorname{tr}\left(T^{\dagger} T\right)+2 m_{O}^{2} \operatorname{tr}\left(O^{\dagger} O\right)-\left(b \mu_{L} H_{u} \tilde{L}_{a}+\text { h.c. }\right)+\left(t_{S} S+\text { h.c. }\right) \\
& +\frac{1}{2} b_{S}\left(S^{2}+\text { h.c. }\right)+b_{T}(\operatorname{tr}(T T)+\text { h.c. })+B_{O}(\operatorname{tr}(O O)+\text { h.c. }) .
\end{aligned}
$$

\footnotetext{
${ }^{1}$ Note that the mass of the charged lepton of flavor a can come from R-symmetry preserving supersymmetry breaking operators [48].
} 
In the R-symmetric case, the lightest eigenvalue of the neutralino mass matrix, written in the basis $\left(\tilde{b}^{0}, \tilde{w}^{0}, \tilde{R}_{d}^{0}, N^{c}\right)$ and $\left(\tilde{S}, \tilde{T}^{0}, \tilde{H}_{u}^{0}, \nu_{e}\right)$, provides a tree level Dirac neutrino mass, which can be written as [50]

$$
m_{\nu_{e}}^{D}=\frac{v^{3} \sin \beta f g \lambda_{T}}{\sqrt{2} \gamma M_{1}^{D} M_{2}^{D}}\left(M_{2}^{D}-M_{1}^{D}\right)
$$

where $M_{1}^{D}, M_{2}^{D}$ stands for Dirac bino and wino masses respectively, $\gamma=\mu_{u}+\lambda_{S} v_{S}+\lambda_{T} v_{T}, g$ is the $\mathrm{SU}(2)$ gauge coupling, $\tan \beta=\frac{v_{u}}{v_{a}}, v \equiv \sqrt{v_{u}^{2}+v_{a}^{2}}=\frac{\sqrt{2} M_{W}}{g}$. To obtain this particular form in eq. (2.7) we have assumed certain relations involving the parameters and they are

$$
\begin{aligned}
\lambda_{T} & =\tan \theta_{W} \lambda_{S}, \\
M_{R} & =\frac{\sqrt{2} f M_{1}^{D} \tan \beta}{g \tan \theta_{W}} .
\end{aligned}
$$

Therefore, with appropriate choice of parameters one can easily obtain a small tree level Dirac neutrino mass $\sim 0.1 \mathrm{eV}$.

\section{Scalar sector}

In this section we shall mention very briefly about the scalar sector of this particular model. For a detailed discussion we refer the reader to [50]. The lightest CP even scalar mass matrix, in the basis of $\left(H_{u}, \tilde{\nu}, S, T\right)$, provide the CP even Higgs boson. It is remarkable that the neutrino Yukawa coupling $f$ renders a tree level correction to the lightest Higgs boson mass, which we calculate as

$$
M_{h}^{2} \leq M_{z}^{2} \cos ^{2} 2 \beta+f^{2} v^{2} \sin ^{2} 2 \beta
$$

For $f \sim \mathcal{O}(1)$ and for small $\tan \beta$, the tree level ${ }^{2}$ Higgs boson mass can satisfy the present observed value, close to $125 \mathrm{GeV}$ [50]. It is also pertinent to mention that the singlet and the triplet fields provide very important loop corrections to the Higgs boson mass. These contributions can be sizable if the singlet and the triplet couplings $\lambda_{S}$ and $\lambda_{T}$ are large. The dominant radiative corrections to the quartic potential can be written as [54], $\frac{1}{2} \delta \lambda_{u}\left(\left|H_{u}\right|^{2}\right)^{2}, \frac{1}{2} \delta \lambda_{\nu}\left(\left|\tilde{\nu}_{a}\right|^{2}\right)^{2}$ and $\frac{1}{2} \delta \lambda_{3}\left|H_{u}^{0}\right|^{2}\left|\tilde{\nu}_{a}\right|^{2}$, where

$$
\begin{aligned}
\delta \lambda_{u}= & \frac{3 y_{t}^{4}}{16 \pi^{2}} \ln \left(\frac{m_{\tilde{t}_{1}} m_{\tilde{t}_{2}}}{m_{t}^{2}}\right)+\frac{5 \lambda_{T}^{4}}{16 \pi^{2}} \ln \left(\frac{m_{T}^{2}}{v^{2}}\right)+\frac{\lambda_{S}^{4}}{16 \pi^{2}} \ln \left(\frac{m_{S}^{2}}{v^{2}}\right)-\frac{1}{16 \pi^{2}} \frac{\lambda_{S}^{2} \lambda_{T}^{2}}{m_{T}^{2}-m_{S}^{2}} \\
& \left(m_{T}^{2}\left\{\ln \left(\frac{m_{T}^{2}}{v^{2}}\right)-1\right\}-m_{S}^{2}\left\{\ln \left(\frac{m_{S}^{2}}{v^{2}}\right)-1\right\}\right), \\
\delta \lambda_{\nu}= & \frac{3 y_{b}^{4}}{16 \pi^{2}} \ln \left(\frac{m_{\tilde{b}_{1}} m_{\tilde{b}_{2}}}{m_{b}^{2}}\right)+\frac{5 \lambda_{T}^{4}}{16 \pi^{2}} \ln \left(\frac{m_{T}^{2}}{v^{2}}\right)+\frac{\lambda_{S}^{4}}{16 \pi^{2}} \ln \left(\frac{m_{S}^{2}}{v^{2}}\right)-\frac{1}{16 \pi^{2}} \frac{\lambda_{S}^{2} \lambda_{T}^{2}}{m_{T}^{2}-m_{S}^{2}} \\
& \left(m_{T}^{2}\left\{\ln \left(\frac{m_{T}^{2}}{v^{2}}\right)-1\right\}-m_{S}^{2}\left\{\ln \left(\frac{m_{S}^{2}}{v^{2}}\right)-1\right\}\right),
\end{aligned}
$$

\footnotetext{
${ }^{2}$ In this paper we shall not explore such a possibility and concentrate on the region of parameter space where $f \sim \mathcal{O}\left(10^{-4}\right)$, which produces a keV sterile neutrino state.
} 
and finally,

$$
\begin{aligned}
\delta \lambda_{3}= & \frac{5 \lambda_{T}^{4}}{32 \pi^{2}} \ln \left(\frac{m_{T}^{2}}{v^{2}}\right)+\frac{1}{32 \pi^{2}} \lambda_{S}^{4} \ln \left(\frac{m_{S}^{2}}{v^{2}}\right)+\frac{1}{32 \pi^{2}} \frac{\lambda_{S}^{2} \lambda_{T}^{2}}{m_{T}^{2}-m_{S}^{2}} \\
& \left(m_{T}^{2}\left\{\ln \left(\frac{m_{T}^{2}}{v^{2}}\right)-1\right\}-m_{S}^{2}\left\{\ln \left(\frac{m_{S}^{2}}{v^{2}}\right)-1\right\}\right) .
\end{aligned}
$$

Therefore, for large $\lambda_{S}, \lambda_{T} \sim \mathcal{O}(1)$, a $125 \mathrm{GeV}$ Higgs boson mass can easily be accommodated in this model even in the presence of a light stop mass and negligible leftright mixing.

\section{R-symmetry breaking}

Until now we have constrained ourselves in the R-symmetry preserving scenario. Although the R-symmetric case in this regard is interesting and should be explored in much more detail but in our work we pursue the path, where R-symmetry is broken. Recent cosmological observations point towards a vanishingly small vacuum energy or cosmological constant associated with our universe. Spontaneously broken supergravity theory in a hidden sector requires a non zero value of the superpotential in vacuum in order to have this small vacuum energy. As the superpotential carries R-charge of two units $(R[W]=2)$, therefore $\mathrm{R}$-symmetry is broken when the superpotential acquires a non zero vev $\langle W\rangle$. Furthermore, a non zero gravitino mass also requires a non zero $\langle W\rangle$, thereby one can consider the gravitino mass as the order parameter of R-symmetry breaking.

The breaking of R-symmetry has to be communicated to the visible sector and in this context we confine ourselves to the case of anomaly mediation, which plays the role of the messenger of R-symmetry breaking $[48,50]$. Such a scenario generates very small $(\sim$ a few MeV) Majorana gaugino masses and trilinear scalar couplings, $M_{i} \sim \frac{g_{i}^{2}}{16 \pi^{2}} m_{3 / 2}$ and $A_{u / d}=\frac{\hat{\beta}_{h_{u / d}} v_{u / d}}{16 \pi^{2} m_{u / d}} m_{3 / 2}[55,56]$, as long as the gravitino mass is in the range of a few GeV.

In the R-breaking case, the neutralino mass matrix written in the basis $\left(\tilde{b}^{0}, \tilde{S}, \tilde{w}^{0}, \tilde{T}, \tilde{R}_{d}^{0}, \tilde{H}_{u}^{0}, N^{c}, \nu_{e}\right)$, is given by

$$
M_{\chi}^{M}=\left(\begin{array}{cccccccc}
M_{1} & M_{1}^{D} & 0 & 0 & 0 & \frac{g^{\prime} v_{u}}{\sqrt{2}} & 0 & -\frac{g^{\prime} v_{a}}{\sqrt{2}} \\
M_{1}^{D} & 0 & 0 & 0 & \lambda_{S} v_{u} & 0 & M_{R} & 0 \\
0 & 0 & M_{2} & M_{2}^{D} & 0 & -\frac{g v_{u}}{\sqrt{2}} & 0 & \frac{g v_{a}}{\sqrt{2}} \\
0 & 0 & M_{2}^{D} & 0 & \lambda_{T} v_{u} & 0 & 0 & 0 \\
0 & \lambda_{S} v_{u} & 0 & \lambda_{T} v_{u} & 0 & \mu_{u}+\lambda_{S} v_{S}+\lambda_{T} v_{T} & 0 & 0 \\
\frac{g^{\prime} v_{u}}{\sqrt{2}} & 0 & -\frac{g v_{u}}{\sqrt{2}} & 0 & \mu_{u}+\lambda_{S} v_{S}+\lambda_{T} v_{T} & 0 & -f v_{a} & 0 \\
0 & M_{R} & 0 & 0 & 0 & -f v_{a} & 0 & -f v_{u} \\
-\frac{g^{\prime} v_{a}}{\sqrt{2}} & 0 & \frac{g v_{a}}{\sqrt{2}} & 0 & 0 & 0 & -f v_{u} & 0
\end{array}\right) .
$$

An approximate expression for the tree level Majorana neutrino mass is given by [50]

$$
\left(m_{\nu}\right)_{\text {Tree }} \simeq-v^{2} \frac{\left[g \lambda_{T} v^{2}\left(M_{2}^{D}-M_{1}^{D}\right) \sin \beta\right]^{2}}{\left[M_{1} \alpha^{2}+M_{2} \delta^{2}\right]},
$$




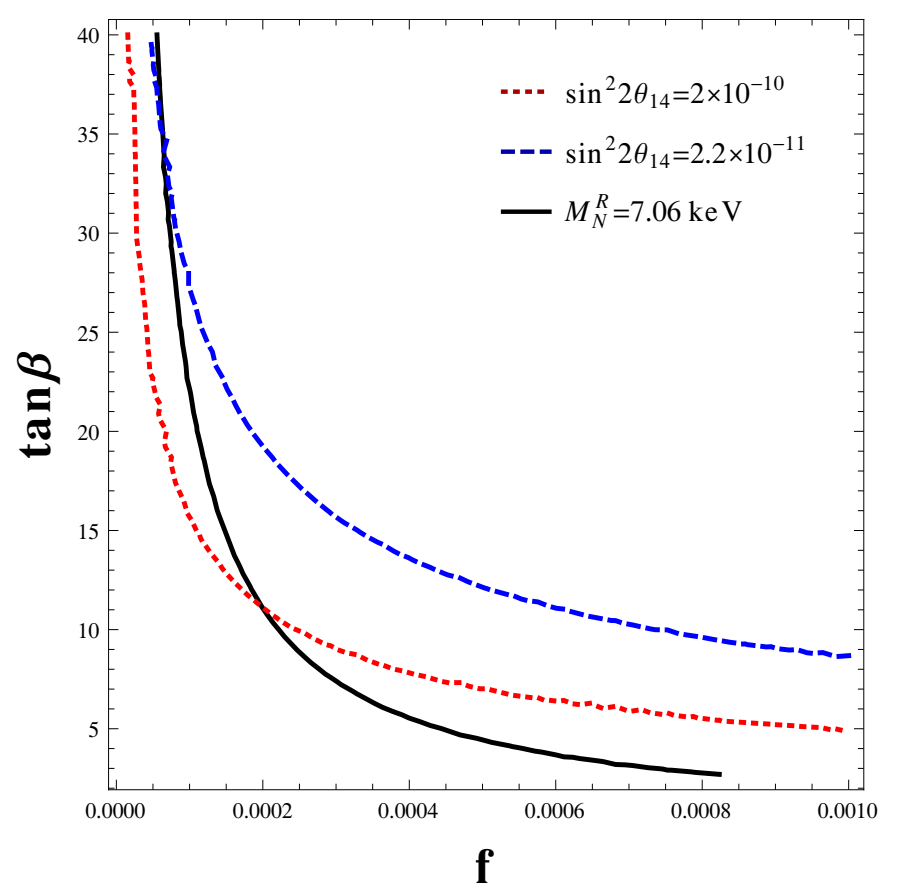

Figure 1. The contour in the black thick line represents a sterile neutrino mass of $7 \mathrm{keV}$. Contours in red (dotted) and blue (dashed) colours show active-sterile mixing $2.2 \times 10^{-11}$ and $2 \times 10^{-10}$ respectively.

where

$$
\begin{aligned}
\alpha & =\frac{2 M_{1}^{D} M_{2}^{D} \gamma \tan \beta}{g \tan \theta_{w}}+\sqrt{2} v^{2} \lambda_{S} \tan \beta\left(M_{1}^{D} \sin ^{2} \beta+M_{2}^{D} \cos ^{2} \beta\right), \\
\delta & =\sqrt{2} M_{1}^{D} v^{2} \lambda_{T} \tan \beta,
\end{aligned}
$$

and $\gamma$ has been defined earlier. Note that the neutrino Yukawa coupling $f$ does not arise in this expression because of our choice in eq. (2.8). Therefore, it is obvious from eq. (4.2) that in order to obtain a small tree level Majorana neutrino mass, we either require a small $\lambda_{T}$ or nearly degenerate Dirac gaugino masses. ${ }^{3}$ In this work we are interested in the sterile neutrino which might play the role of $\mathrm{keV}$ dark matter. From the $8 \times 8$ neutralino mass matrix, the sterile neutrino mass can be approximated as

$$
M_{N}^{R} \simeq M_{1} \frac{2 f^{2} \tan ^{2} \beta}{g^{\prime 2}}
$$

For a wide range of parameters the active-sterile mixing can also be estimated as

$$
\theta_{14}^{2} \simeq \frac{\left(m_{\nu}\right)_{\text {Tree }}}{M_{N}^{R}}
$$

In figure (1), we show in the $(f-\tan \beta)$ plane the contour of sterile neutrino mass fixed

\footnotetext{
${ }^{3} \mathrm{~A}$ detailed discussion on how to fit the light neutrino masses and mixing in this model can be found in $[50]$.
} 


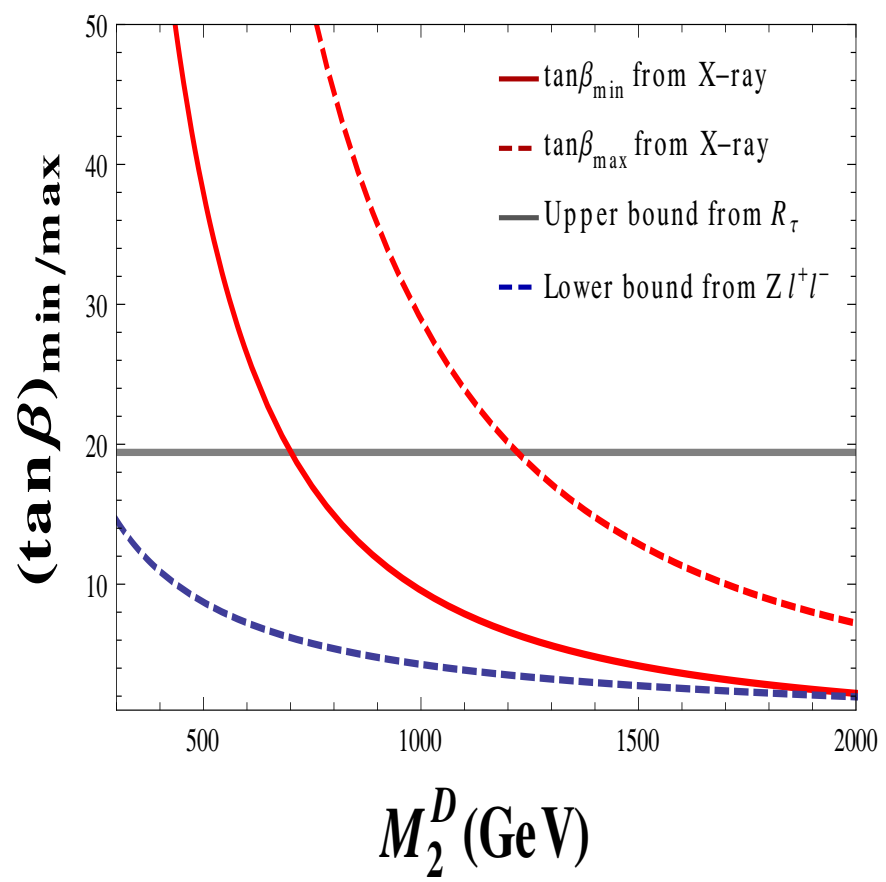

Figure 2. Showing the lower and upper limits of $\tan \beta$ from X-ray analysis as a function of $M_{2}^{D}$ for $\mu_{u}=700 \mathrm{GeV}, m_{3 / 2}=10 \mathrm{GeV}$ and $\epsilon=10^{-4} \mathrm{GeV}$.

at $7.06 \mathrm{keV}$ and also two different contours of $\sin ^{2} 2 \theta_{14}$, fixed at the lower and upper limit at $2.2 \times 10^{-11}$ and $2 \times 10^{-10}$ respectively. We have chosen the gravitino mass, $m_{3 / 2}$ to be $10 \mathrm{GeV}$ and $M_{1}^{D}=900 \mathrm{GeV}$, keeping a degeneracy between the Dirac gaugino masses, $\epsilon \equiv\left(M_{2}^{D}-M_{1}^{D}\right)=10^{-4} \mathrm{GeV}$. We have also fixed $\mu_{u}=750 \mathrm{GeV}, \lambda_{S}=1.1, v_{S}=-0.1 \mathrm{GeV}$ and $v_{T}=0.1 \mathrm{GeV}$.

The sterile neutrino mass contour can be easily explained by looking at eq. (4.4). Similarly from eq. (4.2), eq. (4.4) and eq. (4.5), it is straightforward to show that $\sin ^{2} 2 \theta_{14}$ goes as $\frac{1}{1+\tan ^{2} \beta}$. This means that for smaller $\tan \beta$ one would expect larger mixing angle for fixed values of other parameters. This is also evident from figure 1. Furthermore, for larger Dirac gaugino masses, the active neutrino mass gets reduced (see eq. (4.2)), which also implies a reduction in the active-sterile mixing.

Looking at figure 1 , we observe that the largest value of the active-sterile mixing, required to explain the observed photon line flux at an energy $E \approx 3.5 \mathrm{keV}$, corresponds to the minimum value of $\tan \beta$. In fact, for this particular case shown in figure $1,(\tan \beta)_{\min } \approx$ 11.3. Similarly the smallest active-sterile mixing $\left(\sin ^{2} 2 \theta_{14}=2.2 \times 10^{-11}\right)$ provides the maximum allowed value of $\tan \beta$, which in this case turns out to be $(\tan \beta)_{\max } \approx 33$. In order to obtain an analytical relationship between the lower limit of $\tan \beta$ and $M_{2}^{D}$, we can solve for $\tan \beta$ using eq. (4.5), with $\sin ^{2} 2 \theta_{14}=2 \times 10^{-10}$ and $M_{N}^{R}=7.06 \mathrm{keV}$. This gives rise to

$$
\left(\tan ^{2} \beta\right)_{\min }=\frac{4 v^{2}\left\{g \lambda_{T} v^{2}\left(M_{2}^{D}-M_{1}^{D}\right)\right\}^{2}}{\left(1.4 \times 10^{-15} \mathrm{GeV}\right)\left[M_{1} \alpha^{2}+M_{2} \delta^{\prime 2}\right]}-1,
$$


where

$$
\begin{aligned}
\alpha^{\prime} & \simeq \frac{2\left(M_{2}^{D}\right)^{2} \mu_{u}}{g^{\prime}}+\sqrt{2} v^{2} \lambda_{s} M_{2}^{D}, \\
\delta^{\prime} & \simeq \sqrt{2} M_{2}^{D} v^{2} \lambda_{T} .
\end{aligned}
$$

In a similar way an analytical expression for the upper limit of $\tan \beta$ can also be derived.

Figure 2 shows the lower and upper limits of $\tan \beta$ as a function of $M_{2}^{D}$, for $\mu_{u}=$ $700 \mathrm{GeV}, m_{3 / 2}=10 \mathrm{GeV}$ and $\epsilon=10^{-4} \mathrm{GeV}$. We have fixed $\lambda_{S}$ at the previously mentioned value. The horizontal grey line shows the upper limit on $\tan \beta$ arising from the contribution of the leptonic Yukawa coupling, $f_{\tau} \equiv \lambda_{133}$ to the ratio $R_{\tau} \equiv \Gamma\left(\tau \rightarrow e \bar{\nu}_{e} \nu_{\tau}\right) / \Gamma\left(\tau \rightarrow \mu \bar{\nu}_{\mu} \nu_{\tau}\right)$. The resulting constraint is $f_{\tau}<0.07\left(\frac{m_{\tilde{\tau}_{R}}}{100 \mathrm{GeV}}\right)$ [48] and considering stau mass, close to $280 \mathrm{GeV}$, translates into an upper limit on $\tan \beta \approx 19$. For higher stau mass this upper limit on $\tan \beta$ gets relaxed. The blue dashed line shows the lower bound on $\tan \beta$, as a function of $M_{2}^{D}$, arising from the precision measurements of the deviations in the couplings of the $\mathrm{Z}$ boson to charged leptons [48].

We infer from the above discussions, that in a large region of the parameter space, the lower limit on $\tan \beta$, satisfying the estimated mass and mixing of the sterile neutrino dark matter particle coming from the recent observation of an X-ray line signal at energy $3.5 \mathrm{keV}$ is stronger than the lower limit on $\tan \beta$ coming from the electroweak precision measurements. On the other hand, the upper limit on $\tan \beta$ coming from the X-ray observations becomes stronger than the upper limit arising from the $\tau$ Yukawa coupling contribution to $R_{\tau}$ only for higher values of $M_{2}^{D}$ as shown in figure 2 for specific choices of $\mu_{u}$ and $m_{3 / 2}$. Combining these lower and upper limits on $\tan \beta$ from X-ray observations and measurement of $R_{\tau}$, we can find a range of $M_{2}^{D}$ that is allowed. For smaller values of $\mu_{u}$ and $m_{3 / 2}$, the upper and lower limits of $M_{2}^{D}$ shift to higher values (see eq. (4.6)).

We also observe from figure 1 that the allowed values of $f$ is of the order of $10^{-4}$. Such a small value of $f$, implies negligible extra contribution to the tree level Higgs boson mass. Therefore, to elevate the Higgs boson mass to $125 \mathrm{GeV}$, we have to rely on the loop corrections. Sizable radiative corrections are obtained if $\lambda_{S}, \lambda_{T}$ are large $(\mathcal{O}(1))$ and this would imply nearly degenerate Dirac gaugino masses $\left(\epsilon \sim 10^{-4} \mathrm{GeV}\right)$ in order to have the active-sterile mixing $\sin ^{2} 2 \theta_{14} \sim 10^{-11}$ and a tree level active neutrino mass $\lesssim$ $0.05 \mathrm{eV}$. The other case, which can relax this strong degeneracy between Dirac gaugino masses, corresponds to the case of small $\lambda_{S}, \lambda_{T} \sim \mathcal{O}\left(10^{-4}\right)$, which implies multi-TeV stop to fit the Higgs boson mass. Therefore, this model provides a very interesting possibility where we can connect the Higgs sector with the neutrino sector (both active as well as sterile neutrino).

\section{Right handed neutrino as a keV warm dark matter}

To accommodate sterile neutrino as a warm dark matter candidate, it is very important to make sure that the active sterile mixing is very small [57-62] and within the valid range of different X-ray experiments. A rough bound on the active-sterile mixing can be 
parametrised as [63]

$$
\theta_{14}^{2} \leq 1.8 \times 10^{-5}\left(\frac{1 \mathrm{keV}}{M_{N}^{R}}\right)^{5}
$$

Along with the strict bound coming from different X-ray experiments, the keV sterile neutrino must produce the correct relic density $\Omega_{N} h^{2} \sim 0.1$, in order to identify itself with the warm dark matter. An approximate formula for the relic density of sterile neutrinos, produced in the early universe with negligible lepton asymmetry via non-resonant oscillations with active neutrinos, known as the Dodelson-Widrow (DW) mechanism [64] can be written as [65]

$$
\Omega_{N} h^{2} \sim 0.3\left(\frac{\sin ^{2} 2 \theta_{14}}{10^{-10}}\right)\left(\frac{M_{N}^{R}}{100 \mathrm{keV}}\right)^{2}
$$

where $\Omega_{N}$ is the ratio of the sterile neutrino density to the critical density of the Universe and $h=0.673$.

Different experimental observations have also put lower limits on the mass of the $\mathrm{keV}$ warm dark matter. A very robust bound for fermionic dark matter particles comes from Pauli exclusion principle. By claiming the maximal (Fermi) velocity of the degenerate fermionic gas in the dwarf spheroidal galaxies is less compared to the escape velocity, translates into a lower bound on the sterile neutrino dark matter mass, i.e $M_{N}^{R}>0.41$ $\mathrm{keV}[66,67]$. Model dependent bounds on the mass of the warm dark matter are much more stringent and obtained from analysing Lyman- $\alpha$ experiment $[68,69]$.

In figure 3 we present a scatter plot by scanning the parameter space of our model and also show the compatibility of those points with the current experimental findings. The red circles are the points obtained by varying the parameters as $500 \mathrm{GeV}<\mathrm{M}_{1}^{\mathrm{D}}<$ $1.2 \mathrm{TeV}, 10^{-5}<f<10^{-3}, 2.7<\tan \beta<17,400 \mathrm{GeV}<\mathrm{m}_{\tilde{\mathrm{t}}_{1}, \tilde{\mathrm{t}}_{2}}<1.2 \mathrm{TeV}$, keeping $\epsilon \equiv\left(M_{2}^{D}-M_{1}^{D}\right) \sim 10^{-4} \mathrm{GeV} . \mu_{u}$ and $\lambda_{S}$ are fixed at $750 \mathrm{GeV}$ and 1.1 respectively $\left(\lambda_{T}=\lambda_{S} \tan \theta_{W} \sim 0.6\right)$. All these points respect a Higgs boson mass in between $124.4 \mathrm{GeV}$ and $126.2 \mathrm{GeV}$ avoiding any tachyonic scalar states.

Similar plot can also be generated where $\lambda_{T} \sim 10^{-5}$. Therefore, to fit the Higgs boson mass in that case, one requires $m_{\tilde{t}}>5 \mathrm{TeV}$. However, the degeneracy between $M_{1}^{D}$ and $M_{2}^{D}$ is somewhat lifted where $\epsilon \gtrsim 1 \mathrm{GeV}$.

The horizontal yellow band in figure 3 is ruled out by the Tremaine Gunn bound, which implies $M_{N}^{R}<0.4 \mathrm{keV}[66,67]$. The blue region is excluded by taking into consideration the diffuse X-ray background [70]. Cluster X-ray bound rules out a region in the mass-mixing plane by taking into consideration XMM-Newton observations from the Coma and Virgo clusters [71]. Constraints from the cosmic X-ray background (CXB) rules out the region in red stripes [70]. Chandra observation of M31 [72] rules out the region in grey. The light blue line corresponds to the correct relic density provided by the sterile neutrino warm dark matter via DW mechanism. The light blue region above this line marked as DW is ruled out because of the over abundance of sterile neutrino dark matter. The horizontal and vertical lines show the region in the mass and mixing plane consistent with the observed 3.5 keV X-ray line with more than $3 \sigma$ significance. The black star corresponds to the best fit 


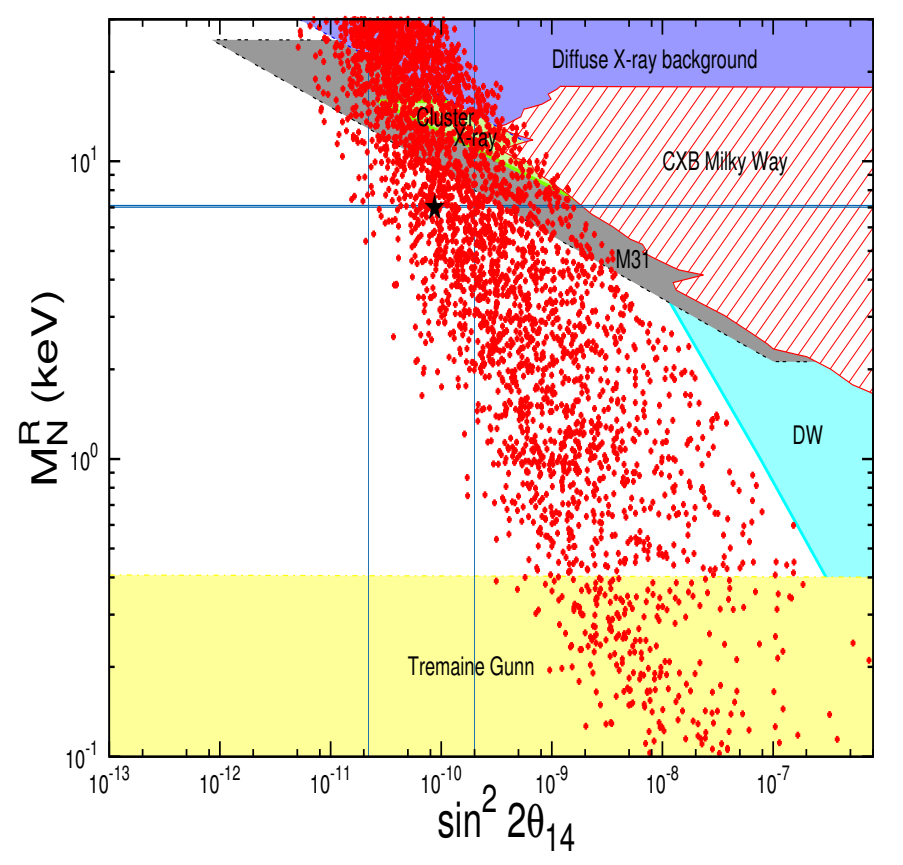

Figure 3. The red (grey) points in the mass-mixing plane are obtained by scanning the parameter space as mentioned in the text. The yellow (light) region is ruled out from the Tremaine Gunn bound [66, 67]. Cosmic X-ray background (CXB) rules out the region in red stripes [70]. Constraints from M31, observed by Chandra rules out the region in grey [72]. The blue region is ruled out from the diffuse X-ray background observations [70]. XMM-Newton observations from Coma and Virgo clusters rule out the region in green. The light blue line represents the $100 \%$ relic density of the sterile neutrino dark matter, produced via DW mechanism. The light blue region above this line leads to over abundance of the sterile neutrino warm dark matter. Finally, the black star represents the central value of the mass and active-sterile mixing, from the $3.5 \mathrm{keV}$ X-ray line observation.

point. It is clearly evident from this figure that such a small mixing is completely in conflict with the DW production mechanism of sterile neutrinos. However, resonant production of sterile neutrinos in the presence of a lepton asymmetry in primordial plasma can be very important and produce correct relic abundance of the $\mathrm{keV}$ sterile neutrinos $[65,73]$. Recent studies have shown that a cosmological lepton asymmetry $L \sim \mathcal{O}\left(10^{-3}\right)$ is capable of producing correct relic density of 0.119 [4]. It was shown in [74-81] that active-sterile neutrino oscillations can themselves create a cosmological lepton number of this magnitude, assuming that the number of sterile neutrinos is negligible to start with. Such a possibility can be easily conceived in our model to generate a large lepton asymmetry.

Let us note in passing that sterile neutrino production in non-standard cosmology with low reheating temperature $(\sim$ a few $\mathrm{MeV})$ has also been discussed in the literature [82-84]. If the universe has undergone inflation and was never reheated to a temperature above a few $\mathrm{MeV}$ then the relic abundance of the sterile neutrinos can be written as

$$
\Omega_{N} h^{2}=10^{-7} d_{\alpha}\left(\frac{\sin ^{2} 2 \theta_{14}}{10^{-10}}\right)\left(\frac{M_{N}^{R}}{10 \mathrm{keV}}\right)\left(\frac{T_{R}}{5 \mathrm{MeV}}\right)^{3},
$$

where $d_{\alpha}=1.13$, assuming that the sterile neutrino couples only with $\nu_{e}$ as in our case. 


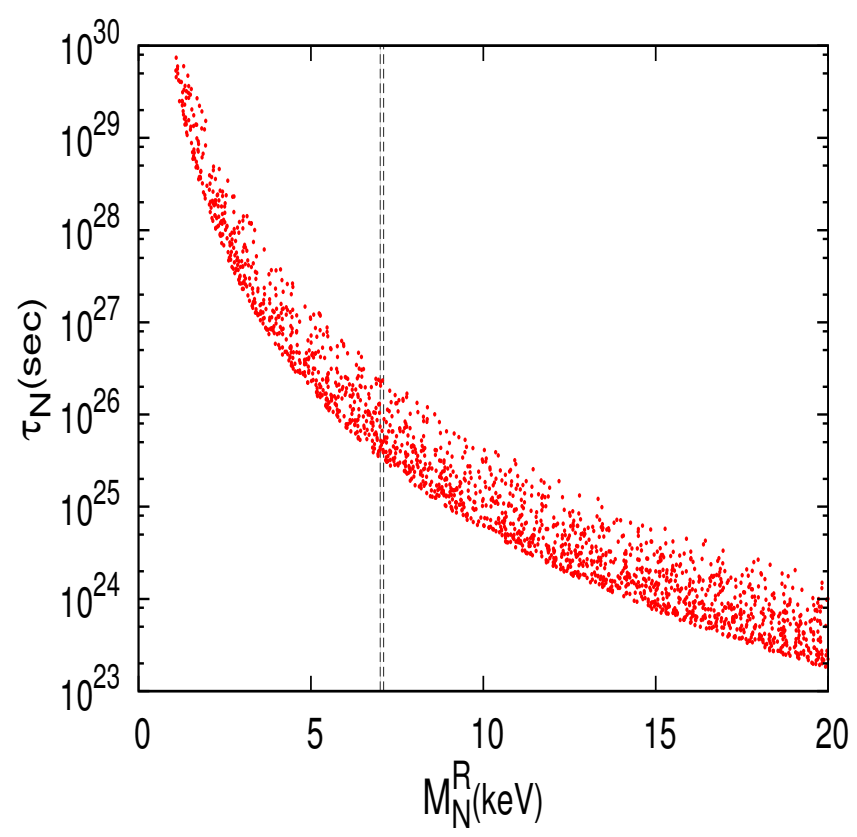

Figure 4. The plot shows the sterile neutrino lifetime as a function of the sterile neutrino mass. The black vertical line represents the $7 \mathrm{keV}$ mass of the sterile neutrino. The red points represent the total life time of the sterile neutrino.

It is obvious from the above expression that for allowed values of $\sin ^{2} 2 \theta_{14}$ and $M_{N}^{R}$ (from the recent $\mathrm{X}$-ray observation) this production mechanism will give rise to severe under abundance of sterile neutrinos.

In our model sterile neutrinos can also be produced non-thermally via the decay of heavier scalar particles. However, a quantitative estimate of the relic density requires a thorough investigation and we postpone the discussion of this method of production for a future work [85].

\subsection{Sterile neutrino decay}

The most dominant decay mode of the sterile neutrino is $N \rightarrow 3 \nu$. The corresponding decay rate for this process is given by [57]

$$
\Gamma_{3 \nu}=8.7 \times 10^{-31} \sec ^{-1}\left(\frac{\sin ^{2} 2 \theta_{14}}{10^{-10}}\right)\left(\frac{M_{N}^{R}}{1 \mathrm{keV}}\right)^{5} .
$$

The principal radiative decay mode of the sterile neutrino which is of concern here is $N \rightarrow \nu \gamma$ and the decay width is

$$
\Gamma_{\nu \gamma}=1.38 \times 10^{-32} \sec ^{-1}\left(\frac{\sin ^{2} 2 \theta_{14}}{10^{-10}}\right)\left(\frac{M_{N}^{R}}{1 \mathrm{keV}}\right)^{5} .
$$

This decay produces a monochromatic photon line at $E_{\gamma}=\frac{M_{N}^{R}}{2}$. From figure (4) we can see that the lifetime of the sterile neutrino is much larger than the age of the universe. 


\section{Gravitino cosmology}

As mentioned earlier, the gravitino mass is the order parameter of R-breaking. If the mass is around a few $\mathrm{GeV}$, it can be a candidate for cold dark matter [86]. In our scenario, the gravitino is an unstable particle and decays to an active/sterile neutrino and a monochromatic photon. The tree level decay mode into an active neutrino final state $\tilde{G} \rightarrow \gamma \nu_{e}$ is suppressed by the very small mixing $U_{\tilde{b} \nu_{e}}\left(\sim 10^{-7}\right)$ between the bino and active neutrino $\nu_{e}$ [87]. Interestingly, in our model the most dominant decay mode of gravitino is into a photon and a sterile neutrino $(\tilde{G} \rightarrow N \gamma)$ and the decay width is given as

$$
\Gamma_{\tilde{G} \rightarrow N \gamma} \sim \frac{\left|U_{\tilde{b} N}\right|^{2} m_{3 / 2}^{3}}{32 \pi M_{P}^{2}},
$$

where $U_{\tilde{b} N}$ is the bino sterile neutrino mixing angle. Because of the presence of the term $M_{R} \hat{N}^{c} \hat{S}$ in the superpotential and the bino Dirac mass term in the Lagrangian, the tree level bino sterile neutrino mixing is not strongly suppressed $\left(\sim 10^{-2}\right)$.

For the sake of completeness, let us mention that at the one loop level the decay $\tilde{G} \rightarrow$ $\gamma \nu_{e}$ occurs [88-91] via trilinear R-parity violating coupling $\lambda_{133}^{\prime}$ which we have identified with the bottom Yukawa coupling. We have checked that this process is also suppressed compared to the tree level decay $\tilde{G} \rightarrow N \gamma$. The one-loop contribution to the decay $\tilde{G} \rightarrow N \gamma$ is negligible because of small active-sterile mixing.

Taking into account the most dominant decay mode of the gravitino in the sterile neutrino plus photon final state, for a $10 \mathrm{GeV}$ gravitino mass, the lifetime is close to $10^{15}$ sec. Therefore, to satisfy the experimental constraints coming from the diffuse photon background, one has to consider a scenario where the gravitino density is very much diluted. In order to provide a quantitative analysis we note that for a gravitino of mass $10 \mathrm{GeV}$ the limit on the diffuse photon flux is around $6.89 \times 10^{-7} \mathrm{GeVcm}^{-2} \mathrm{sec}^{-1}$ [92]. This can be translated into a bound on the gravitino relic density and we find

$$
\Omega_{3 / 2} h^{2}<4.34 \times 10^{-13}\left(\frac{10^{-2}}{U_{\tilde{b} N}}\right)^{2},
$$

for a $10 \mathrm{GeV}$ gravitino. Note that this bound depends strongly on the mass of the gravitino and will get relaxed for a smaller gravitino mass. To satisfy such a strong bound on the gravitino relic density, one must account for a very low reheating temperature. If the reheating temperature is above the SUSY scale, the gravitino relic density would be too large [93]. Therefore, the reheating temperature must lie much below the SUSY threshold.

Following [43], we see that if the reheating temperature is below the SUSY threshold, the gravitinos are produced by thermal scattering with neutrinos and bottom quarks. Using the results of [43] for production of gravitinos, we obtain an upper bound on the reheating temperature for a $10 \mathrm{GeV}$ gravitino as

$$
T_{R}<127\left(\frac{v_{a}}{30 \mathrm{GeV}}\right)^{2 / 7}\left(\frac{m_{\tilde{b}}}{500 \mathrm{GeV}}\right)^{4 / 7}\left(\frac{10^{-2}}{U_{\tilde{b} N}}\right)^{2 / 7} \mathrm{GeV} .
$$


Such a low reheating temperature might have important implications in the context of different baryogenesis and leptogenesis scenarios.

\section{Conclusion}

Recent observation of a weak X-ray line around $E_{\gamma}=3.5 \mathrm{keV}$ by XMM-Newton telescope coming from Andromeda galaxy and various galaxy clusters have been studied in the light of a $\mathrm{U}(1)_{R}$-lepton number model, with a single right handed neutrino. We have shown explicitly that a sterile neutrino of mass about $7 \mathrm{keV}$ and with appropriate active-sterile mixing can easily be obtained in our model. We briefly mention different production mechanisms of the sterile neutrino.

Allowed ranges of the mass and mixing helped us to put bounds on $\tan \beta$ as a function of the Dirac wino mass $M_{2}^{D}$. Combining these bounds with the limits coming from the measurements of the $\tau$ Yukawa coupling contribution to the ratio $R_{\tau} \equiv \Gamma\left(\tau \rightarrow e \bar{\nu}_{e} \nu_{\tau}\right) / \Gamma(\tau \rightarrow$ $\mu \bar{\nu}_{\mu} \nu_{\tau}$ ), one obtains strong upper and lower bounds on $M_{2}^{D}$.

In addition, we have also discussed the Higgs sector briefly and pointed out different possibilities to have a Higgs boson mass around $125 \mathrm{GeV}$. Finally, gravitino is the LSP in our model with a mass about a few $\mathrm{GeV}$ and gravitino mass is the order parameter of $\mathrm{R}$ symmetry breaking. The gravitino can decay into a photon plus active or sterile neutrino. Therefore, we have also presented a short discussion on the cosmological implications of the gravitino. We have taken into account the most robust constraint coming from the diffuse photon background, which readily puts a very stringent bound on the gravitino relic density. This eventually imposes an upper limit $(\lesssim 130 \mathrm{GeV})$ on the reheating temperature of the universe.

\section{Acknowledgments}

We thank Kevork N. Abazajian and George G Raffelt for helpful discussions. S.C would also like to thank the Council of Scientific and Industrial Research (CSIR), Government of India for financial support obtained as a Senior Research Fellow.

Open Access. This article is distributed under the terms of the Creative Commons Attribution License (CC-BY 4.0), which permits any use, distribution and reproduction in any medium, provided the original author(s) and source are credited.

\section{References}

[1] E. Bulbul et al., Detection of an unidentified emission line in the stacked X-ray spectrum of galaxy clusters, Astrophys. J. 789 (2014) 13 [arXiv:1402.2301] [INSPIRE].

[2] A. Boyarsky, O. Ruchayskiy, D. Iakubovskyi and J. Franse, An unidentified line in X-ray spectra of the Andromeda galaxy and Perseus galaxy cluster, arXiv:1402.4119 [INSPIRE].

[3] H. Ishida, K.S. Jeong and F. Takahashi, 7 keV sterile neutrino dark matter from split flavor mechanism, Phys. Lett. B 732 (2014) 196 [arXiv:1402.5837] [INSPIRE]. 
[4] K.N. Abazajian, Resonantly-produced 7 keV sterile neutrino dark matter models and the properties of Milky Way satellites, Phys. Rev. Lett. 112 (2014) 161303 [arXiv:1403.0954] [INSPIRE].

[5] K.P. Modak, $3.5 \mathrm{keV}$ X-ray line signal from decay of right-handed neutrino due to transition magnetic moment, arXiv:1404.3676 [INSPIRE].

[6] J.M. Cline, Y. Farzan, Z. Liu, G.D. Moore and W. Xue, $3.5 \mathrm{keV}$ X-rays as the "21 cm line" of dark atoms and a link to light sterile neutrinos, Phys. Rev. D 89 (2014) 121302 [arXiv: 1404.3729] [INSPIRE].

[7] J. Barry, J. Heeck and W. Rodejohann, Sterile neutrinos and right-handed currents in KATRIN, JHEP 07 (2014) 081 [arXiv: 1404.5955] [INSPIRE].

[8] D.J. Robinson and Y. Tsai, A dynamical framework for KeV Dirac neutrino warm dark matter, Phys. Rev. D 90 (2014) 045030 [arXiv:1404.7118] [INSPIRE].

[9] T. Higaki, K.S. Jeong and F. Takahashi, The 7 keV axion dark matter and the X-ray line signal, Phys. Lett. B 733 (2014) 25 [arXiv:1402.6965] [INSPIRE].

[10] J. Jaeckel, J. Redondo and A. Ringwald, A $3.55 \mathrm{keV}$ hint for decaying axion-like particle dark matter, Phys. Rev. D 89 (2014) 103511 [arXiv:1402.7335] [INSPIRE].

[11] H.M. Lee, S.C. Park and W.-I. Park, Cluster X-ray line at $3.5 \mathrm{keV}$ from axion-like dark matter, Eur. Phys. J. C 74 (2014) 3062 [arXiv:1403.0865] [INSPIRE].

[12] J.P. Conlon and F.V. Day, $3.55 \mathrm{keV}$ photon lines from axion to photon conversion in the Milky Way and M31, arXiv:1404.7741 [INSPIRE].

[13] J.-C. Park, S.C. Park and K. Kong, X-ray line signal from 7 keV axino dark matter decay, Phys. Lett. B 733 (2014) 217 [arXiv:1403.1536] [INSPIRE].

[14] K.-Y. Choi and O. Seto, X-ray line signal from decaying axino warm dark matter, Phys. Lett. B 735 (2014) 92 [arXiv:1403.1782] [InSPIRE].

[15] S.P. Liew, Axino dark matter in light of an anomalous X-ray line, JCAP 05 (2014) 044 [arXiv: 1403.6621] [INSPIRE].

[16] S. Baek and H. Okada, 7 keV dark matter as X-ray line signal in radiative neutrino model, arXiv:1403.1710 [INSPIRE].

[17] H. Okada and T. Toma, $3.55 \mathrm{keV}$ X-ray line signal from excited dark matter in radiative neutrino model, Phys. Lett. B 737 (2014) 162 [arXiv:1404.4795] [INSPIRE].

[18] N.E. Bomark and L. Roszkowski, $3.5 \mathrm{keV}$ x-ray line from decaying gravitino dark matter, Phys. Rev. D 90 (2014) 011701 [arXiv: 1403.6503] [INSPIRE].

[19] S.V. Demidov and D.S. Gorbunov, SUSY in the sky or a keV signature of sub-GeV gravitino dark matter, arXiv: 1404.1339 [INSPIRE].

[20] C. Kolda and J. Unwin, X-ray lines from R-parity violating decays of ke $V$ sparticles, Phys. Rev. D 90 (2014) 023535 [arXiv: 1403.5580] [INSPIRE].

[21] E. Dudas, L. Heurtier and Y. Mambrini, Generating X-ray lines from annihilating dark matter, Phys. Rev. D 90 (2014) 035002 [arXiv: 1404.1927] [INSPIRE].

[22] F.S. Queiroz and K. Sinha, The poker face of the Majoron dark matter model: LUX to keV line, Phys. Lett. B 735 (2014) 69 [arXiv:1404.1400] [InSPIRE]. 
[23] Z. Kang, P. Ko, T. Li and Y. Liu, Natural X-ray lines from the low scale supersymmetry breaking, arXiv: 1403.7742 [INSPIRE].

[24] ATLAS collaboration, Observation of a new particle in the search for the standard model Higgs boson with the ATLAS detector at the LHC, Phys. Lett. B 716 (2012) 1 [arXiv:1207.7214] [INSPIRE].

[25] CMS collaboration, Observation of a new boson at a mass of $125 \mathrm{GeV}$ with the CMS experiment at the LHC, Phys. Lett. B 716 (2012) 30 [arXiv:1207.7235] [INSPIRE].

[26] CMS collaboration, Search for supersymmetry in hadronic final states with missing transverse energy using the variables $\alpha_{T}$ and b-quark multiplicity in pp collisions at $\sqrt{s}=8$ TeV, Eur. Phys. J. C 73 (2013) 2568 [arXiv:1303.2985] [InSPIRE].

[27] ATLAS collaboration, Search for new phenomena in final states with large jet multiplicities and missing transverse momentum at $\sqrt{s}=8 \mathrm{TeV}$ proton-proton collisions using the ATLAS experiment, JHEP 10 (2013) 130 [arXiv:1308.1841] [INSPIRE].

[28] P. Fayet, Supersymmetry and weak, electromagnetic and strong interactions, Phys. Lett. B 64 (1976) 159 [inSPIRE].

[29] L.J. Hall, Alternative low-energy supersymmetry, Mod. Phys. Lett. A 5 (1990) 467 [INSPIRE].

[30] L.J. Hall and L. Randall, U(1) - R symmetric supersymmetry, Nucl. Phys. B 352 (1991) 289 [INSPIRE].

[31] A.E. Nelson, N. Rius, V. Sanz and M. Ünsal, The minimal supersymmetric model without a $\mu$ term, JHEP 08 (2002) 039 [hep-ph/0206102] [INSPIRE].

[32] P.J. Fox, A.E. Nelson and N. Weiner, Dirac gaugino masses and supersoft supersymmetry breaking, JHEP 08 (2002) 035 [hep-ph/0206096] [INSPIRE].

[33] Z. Chacko, P.J. Fox and H. Murayama, Localized supersoft supersymmetry breaking, Nucl. Phys. B 706 (2005) 53 [hep-ph/0406142] [INSPIRE].

[34] S.Y. Choi, M. Drees, A. Freitas and P.M. Zerwas, Testing the Majorana nature of gluinos and neutralinos, Phys. Rev. D 78 (2008) 095007 [arXiv:0808.2410] [INSPIRE].

[35] G.D. Kribs, E. Poppitz and N. Weiner, Flavor in supersymmetry with an extended R-symmetry, Phys. Rev. D 78 (2008) 055010 [arXiv:0712.2039] [INSPIRE].

[36] K. Benakli and M.D. Goodsell, Dirac gauginos in general gauge mediation, Nucl. Phys. B 816 (2009) 185 [arXiv:0811.4409] [INSPIRE].

[37] A. Kumar, D. Tucker-Smith and N. Weiner, Neutrino mass, sneutrino dark matter and signals of lepton flavor violation in the MRSSM, JHEP 09 (2010) 111 [arXiv:0910.2475] [INSPIRE].

[38] B.A. Dobrescu and P.J. Fox, Uplifted supersymmetric Higgs region, Eur. Phys. J. C 70 (2010) 263 [arXiv:1001.3147] [INSPIRE].

[39] K. Benakli, M.D. Goodsell and A.-K. Maier, Generating $\mu$ and B $\mu$ in models with Dirac gauginos, Nucl. Phys. B 851 (2011) 445 [arXiv:1104.2695] [InSPIRE].

[40] K. Benakli, Dirac gauginos: a user manual, Fortsch. Phys. 59 (2011) 1079 [arXiv: 1106.1649] [INSPIRE].

[41] R. Davies and M. McCullough, Small neutrino masses due to R-symmetry breaking for a small cosmological constant, Phys. Rev. D 86 (2012) 025014 [arXiv:1111.2361] [INSPIRE]. 
[42] R. Davies, J. March-Russell and M. McCullough, A supersymmetric one Higgs doublet model, JHEP 04 (2011) 108 [arXiv: 1103.1647] [INSPIRE].

[43] C. Frugiuele and T. Gregoire, Making the sneutrino a Higgs with a $\mathrm{U}(1)_{R}$ lepton number, Phys. Rev. D 85 (2012) 015016 [arXiv:1107.4634] [InSPIRE].

[44] E. Bertuzzo, C. Frugiuele, T. Gregoire and E. Ponton, Dirac gauginos, $R$ symmetry and the $125 \mathrm{GeV}$ Higgs, arXiv: 1402.5432 [INSPIRE].

[45] E. Bertuzzo and C. Frugiuele, Fitting neutrino physics with a $\mathrm{U}(1)_{R}$ lepton number, JHEP 05 (2012) 100 [arXiv:1203.5340] [INSPIRE].

[46] K. Benakli, M.D. Goodsell and F. Staub, Dirac gauginos and the $125 \mathrm{GeV}$ Higgs, JHEP 06 (2013) 073 [arXiv:1211.0552] [INSPIRE].

[47] F. Riva, C. Biggio and A. Pomarol, Is the 125 GeV Higgs the superpartner of a neutrino?, JHEP 02 (2013) 081 [arXiv: 1211.4526] [INSPIRE].

[48] C. Frugiuele, T. Gregoire, P. Kumar and E. Ponton, ' $L=R$ ' $-\mathrm{U}(1)_{R}$ as the origin of leptonic ' $R P V$ ', JHEP 03 (2013) 156 [arXiv:1210.0541] [INSPIRE].

[49] C. Frugiuele, T. Gregoire, P. Kumar and E. Ponton, ' $L=R$ ' $-\mathrm{U}(1)_{R}$ lepton number at the LHC, JHEP 05 (2013) 012 [arXiv: 1210.5257] [INSPIRE].

[50] S. Chakraborty and S. Roy, Higgs boson mass, neutrino masses and mixing and keV dark matter in an $\mathrm{U}(1)_{R}$-lepton number model, JHEP 01 (2014) 101 [arXiv:1309.6538] [INSPIRE].

[51] E. Dudas, M. Goodsell, L. Heurtier and P. Tziveloglou, Flavour models with Dirac and fake gluinos, Nucl. Phys. B 884 (2014) 632 [arXiv:1312.2011] [InSPIRE].

[52] H. Beauchesne and T. Gregoire, Electroweak precision measurements in supersymmetric models with a $\mathrm{U}(1)_{R}$ lepton number, JHEP 05 (2014) 051 [arXiv: 1402.5403] [INSPIRE].

[53] K. Benakli, M. Goodsell, F. Staub and W. Porod, The constrained minimal Dirac gaugino supersymmetric standard model, Phys. Rev. D 90 (2014) 045017 [arXiv:1403.5122] [INSPIRE].

[54] G. Bélanger, K. Benakli, M. Goodsell, C. Moura and A. Pukhov, Dark matter with Dirac and Majorana gaugino masses, JCAP 08 (2009) 027 [arXiv: 0905.1043] [INSPIRE].

[55] T. Gherghetta, G.F. Giudice and J.D. Wells, Phenomenological consequences of supersymmetry with anomaly induced masses, Nucl. Phys. B 559 (1999) 27 [hep-ph/9904378] [INSPIRE].

[56] D.K. Ghosh, A. Kundu, P. Roy and S. Roy, Characteristic wino signals in a linear collider from anomaly mediated supersymmetry breaking, Phys. Rev. D 64 (2001) 115001 [hep-ph/0104217] [INSPIRE].

[57] K. Abazajian, G.M. Fuller and W.H. Tucker, Direct detection of warm dark matter in the X-ray, Astrophys. J. 562 (2001) 593 [astro-ph/0106002] [INSPIRE].

[58] A. Boyarsky, A. Neronov, O. Ruchayskiy, M. Shaposhnikov and I. Tkachev, Where to find a dark matter sterile neutrino?, Phys. Rev. Lett. 97 (2006) 261302 [astro-ph/0603660] [INSPIRE].

[59] A. Boyarsky, A. Neronov, O. Ruchayskiy and M. Shaposhnikov, Restrictions on parameters of sterile neutrino dark matter from observations of galaxy clusters, Phys. Rev. D 74 (2006) 103506 [astro-ph/0603368] [INSPIRE]. 
[60] M. Loewenstein, A. Kusenko and P.L. Biermann, New limits on sterile neutrinos from Suzaku observations of the Ursa Minor dwarf spheroidal galaxy,

Astrophys. J. 700 (2009) 426 [arXiv:0812.2710] [INSPIRE].

[61] S. Riemer-Sorensen, K. Pedersen, S.H. Hansen and H. Dahle, Probing the nature of dark matter with cosmic X-rays: constraints from dark blobs and grating spectra of galaxy clusters, Phys. Rev. D 76 (2007) 043524 [astro-ph/0610034] [INSPIRE].

[62] R.S. Hundi and S. Roy, Constraints on composite Dirac neutrinos from observations of galaxy clusters, Phys. Lett. B 702 (2011) 228 [arXiv: 1105.0291] [INSPIRE].

[63] A. Boyarsky, O. Ruchayskiy and M. Shaposhnikov, The role of sterile neutrinos in cosmology and astrophysics, Ann. Rev. Nucl. Part. Sci. 59 (2009) 191 [arXiv:0901.0011] [INSPIRE].

[64] S. Dodelson and L.M. Widrow, Sterile-neutrinos as dark matter, Phys. Rev. Lett. 72 (1994) 17 [hep-ph/9303287] [INSPIRE].

[65] K. Abazajian, G.M. Fuller and M. Patel, Sterile neutrino hot, warm and cold dark matter, Phys. Rev. D 64 (2001) 023501 [astro-ph/0101524] [INSPIRE].

[66] S. Tremaine and J.E. Gunn, Dynamical role of light neutral leptons in cosmology, Phys. Rev. Lett. 42 (1979) 407 [INSPIRE].

[67] A. Boyarsky, O. Ruchayskiy and D. Iakubovskyi, A lower bound on the mass of dark matter particles, JCAP 03 (2009) 005 [arXiv:0808.3902] [INSPIRE].

[68] U. Seljak, A. Makarov, P. McDonald and H. Trac, Can sterile neutrinos be the dark matter?, Phys. Rev. Lett. 97 (2006) 191303 [astro-ph/0602430] [INSPIRE].

[69] A. Boyarsky, J. Lesgourgues, O. Ruchayskiy and M. Viel, Lyman- $\alpha$ constraints on warm and on warm-plus-cold dark matter models, JCAP 05 (2009) 012 [arXiv:0812.0010] [INSPIRE].

[70] K.N. Abazajian, M. Markevitch, S.M. Koushiappas and R.C. Hickox, Limits on the radiative decay of sterile neutrino dark matter from the unresolved cosmic and soft X-ray backgrounds, Phys. Rev. D 75 (2007) 063511 [astro-ph/0611144] [INSPIRE].

[71] A. Boyarsky, A. Neronov, O. Ruchayskiy and M. Shaposhnikov, Constraints on sterile neutrino as a dark matter candidate from the diffuse $x$-ray background, Mon. Not. Roy. Astron. Soc. 370 (2006) 213 [astro-ph/0512509] [INSPIRE].

[72] C.R. Watson, J.F. Beacom, H. Yuksel and T.P. Walker, Direct X-ray constraints on sterile neutrino warm dark matter, Phys. Rev. D 74 (2006) 033009 [astro-ph/0605424] [INSPIRE].

[73] X.-D. Shi and G.M. Fuller, A new dark matter candidate: nonthermal sterile neutrinos, Phys. Rev. Lett. 82 (1999) 2832 [astro-ph/9810076] [INSPIRE].

[74] R. Foot, M.J. Thomson and R.R. Volkas, Large neutrino asymmetries from neutrino oscillations, Phys. Rev. D 53 (1996) 5349 [hep-ph/9509327] [INSPIRE].

[75] X.-D. Shi, Chaotic amplification of neutrino chemical potentials by neutrino oscillations in Big Bang nucleosynthesis, Phys. Rev. D 54 (1996) 2753 [astro-ph/9602135] [INSPIRE].

[76] R. Foot and R.R. Volkas, Studies of neutrino asymmetries generated by ordinary sterile neutrino oscillations in the early universe and implications for big bang nucleosynthesis bounds, Phys. Rev. D 55 (1997) 5147 [hep-ph/9610229] [INSPIRE].

[77] R. Foot and R.R. Volkas, Reconciling sterile neutrinos with big bang nucleosynthesis, Phys. Rev. Lett. 75 (1995) 4350 [hep-ph/9508275] [INSPIRE]. 
[78] C.T. Kishimoto and G.M. Fuller, Lepton number-driven sterile neutrino production in the early universe, Phys. Rev. D 78 (2008) 023524 [arXiv:0802.3377] [INSPIRE].

[79] A.D. Dolgov, Neutrinos in the early universe, Sov. J. Nucl. Phys. 33 (1981) 700 [Yad. Fiz. 33 (1981) 1309] [INSPIRE].

[80] R. Barbieri and A. Dolgov, Bounds on sterile-neutrinos from nucleosynthesis, Phys. Lett. B 237 (1990) 440 [INSPIRE].

[81] K. Enqvist, K. Kainulainen and J. Maalampi, Neutrino asymmetry and oscillations in the early universe, Phys. Lett. B 244 (1990) 186 [INSPIRE].

[82] G. Gelmini, S. Palomares-Ruiz and S. Pascoli, Low reheating temperature and the visible sterile neutrino, Phys. Rev. Lett. 93 (2004) 081302 [astro-ph/0403323] [INSPIRE].

[83] C.E. Yaguna, Sterile neutrino production in models with low reheating temperatures, JHEP 06 (2007) 002 [arXiv:0706.0178] [INSPIRE].

[84] G. Gelmini, E. Osoba, S. Palomares-Ruiz and S. Pascoli, MeV sterile neutrinos in low reheating temperature cosmological scenarios, JCAP 10 (2008) 029 [arXiv:0803.2735] [INSPIRE].

[85] S. Chakraborty and S. Roy, New production mode for sterile neutrinos in $\mathrm{U}(1)_{R}$-lepton number model, work in progress.

[86] W. Buchmüller, L. Covi, K. Hamaguchi, A. Ibarra and T. Yanagida, Gravitino dark matter in R-parity breaking vacua, JHEP 03 (2007) 037 [hep-ph/0702184] [INSPIRE].

[87] F. Takayama and M. Yamaguchi, Gravitino dark matter without R-parity, Phys. Lett. B 485 (2000) 388 [hep-ph/0005214] [INSPIRE].

[88] S. Borgani, A. Masiero and M. Yamaguchi, Light gravitinos as mixed dark matter, Phys. Lett. B 386 (1996) 189 [hep-ph/9605222] [INSPIRE].

[89] N.-E. Bomark, S. Lola, P. Osland and A.R. Raklev, Gravitino dark matter and the flavour structure of R-violating operators, Phys. Lett. B 677 (2009) 62 [arXiv:0811.2969] [INSPIRE].

[90] N.-E. Bomark, S. Lola, P. Osland and A.R. Raklev, Photon, neutrino and charged particle spectra from R-violating gravitino decays, Phys. Lett. B 686 (2010) 152 [arXiv:0911.3376] [INSPIRE].

[91] B. Bajc, T. Enkhbat, D.K. Ghosh, G. Senjanović and Y. Zhang, MSSM in view of PAMELA and Fermi-LAT, JHEP 05 (2010) 048 [arXiv:1002.3631] [INSPIRE].

[92] H. Yuksel and M.D. Kistler, Circumscribing late dark matter decays model independently, Phys. Rev. D 78 (2008) 023502 [arXiv:0711.2906] [INSPIRE].

[93] M. Bolz, A. Brandenburg and W. Buchmüller, Thermal production of gravitinos, Nucl. Phys. B 606 (2001) 518 [Erratum ibid. B 790 (2008) 336] [hep-ph/0012052] [INSPIRE]. 